\title{
Imaging a positronium cloud in a 1 Tesla
}

Antoine Camper ${ }^{1, *}$, Stefano Aghion ${ }^{2,3}$, Claude Amsler ${ }^{4}$, Massimiliano Antonello ${ }^{3,5}$, Alexander Belov ${ }^{6}$, Germano Bonomi ${ }^{7,8}$, Roberto Brusa ${ }^{9,10}$, Massimo Caccia ${ }^{3,5}$, Ruggero Caravita $^{1}$, Fabrizio Castelli ${ }^{3,11}$, Giovanni Cerchiari ${ }^{12}$, Daniel Comparat ${ }^{13}$, Giovanni Consolati $^{2,3}$, Andrea Demetrio ${ }^{14}$, Lea Di Noto ${ }^{15,16}$, Michael Doser ${ }^{1}$, Craig Evans ${ }^{2,3}$, Mattia Fanì $^{1,15,16}$, Rafael Ferragut ${ }^{2,3}$, Julian Fesel $^{1}$, Andrea Fontana ${ }^{8}$, Sebastian Gerber ${ }^{1}$, Marco Giammarchi $^{3}$, Angela Gligorova ${ }^{4}$, Francesco Guatieri ${ }^{9,10}$, Philip Hackstock ${ }^{4}$, Stefan Haider $^{1}$, Alexander Hinterberger ${ }^{1}$, Helga Holmestad $^{17}$, Alban Kellerbauer ${ }^{12}$, Olga Khalidova $^{1}$, Daniel Krasnický ${ }^{16}$, Vittorio Lagomarsino ${ }^{15,16}$, Pierre Lansonneur ${ }^{18}$, Patrice Lebrun $^{18}$, Chloé Malbrunot ${ }^{1,4}$, Sebastiano Mariazzi ${ }^{9,10}$, Johann Marton ${ }^{4}$, Viktor Matveev ${ }^{6,19}$, Simon Müller ${ }^{14}$, Giancarlo Nebbia ${ }^{20}$, Patrick Nedelec ${ }^{18}$, Markus Oberthaler ${ }^{14}$, Davide Pagano ${ }^{7,8}$, Luca Penasa ${ }^{9,10}$, Vojtech Petracek ${ }^{21}$, Francesco Prelz ${ }^{3}$, Marco Prevedelli ${ }^{22}$, Benjamin Rienaecker ${ }^{1}$, Jacques Robert ${ }^{13}$, Ole Røhne ${ }^{17}$, Alberto Rotondi ${ }^{8,23}$, Heidi Sandaker ${ }^{17}$, Romualdo Santoro ${ }^{3,5}$, Lillian Smestad ${ }^{1,24}$, Fiodor Sorrentino ${ }^{15,16}$, Gemma Testera $^{16}$, Ingmari Tietje ${ }^{1}$, Milena Vujanovic ${ }^{1}$, Eberhard Widmann ${ }^{4}$, Pauline Yzombard ${ }^{12}$, Christian Zimmer ${ }^{1,12,25}$, Johann Zmeskal ${ }^{4}$, and Nicola Zurlo ${ }^{8,26}$

${ }^{1}$ Physics Department, CERN, 1211 Geneva 23, Switzerland

${ }^{2}$ Politecnico of Milano, Piazza Leonardo da Vinci 32, 20133 Milano, Italy

${ }^{3}$ INFN Milano, via Celoria 16, 20133, Milano, Italy

${ }^{4}$ Stefan Meyer Institute for Subatomic Physics, Austrian Academy of Sciences, Boltzmanngasse 3, 1090 Vienna, Austria

${ }^{5}$ Department of Science, University of Insubria, Via Valleggio 11, 22100 Como, Italy

${ }^{6}$ Institute for Nuclear Research of the Russian Academy of Science, Moscow 117312, Russia

${ }^{7}$ Department of Mechanical and Industrial Engineering, University of Brescia, via Branze 38, 25123

Brescia, Italy

${ }^{8}$ INFN Pavia, via Bassi 6, 27100 Pavia, Italy

${ }^{9}$ Department of Physics, University of Trento, via Sommarive 14, 38123 Povo, Trento, Italy

${ }^{10}$ TIFPA/INFN Trento, via Sommarive 14, 38123 Povo, Trento, Italy

${ }^{11}$ Department of Physics, University of Milano, via Celoria 16, 20133 Milano, Italy

${ }^{12}$ Max Planck Institute for Nuclear Physics, Saupfercheckweg 1, 69117 Heidelberg, Germany

${ }^{13}$ Laboratoire Aimé Cotton, Université Paris-Sud, ENS Cachan, CNRS, Université Paris-Saclay, 91405 Orsay Cedex, France

${ }^{14}$ Kirchhoff-Institute for Physics, Heidelberg University, Im Neuenheimer Feld 227, 69120

Heidelberg, Germany

${ }^{15}$ Department of Physics, University of Genova, via Dodecaneso 33, 16146 Genova, Italy

${ }^{16}$ INFN Genova, via Dodecaneso 33, 16146 Genova, Italy

${ }^{17}$ Department of Physics, University of Oslo, Semælands vei 24, 0371 Oslo, Norway

${ }^{18}$ Institute of Nuclear Physics, CNRS/IN2p3, University of Lyon 1, 69622 Villeurbanne, France

${ }^{19}$ Joint Institute for Nuclear Research, 141980 Dubna, Russia

${ }^{20}$ INFN Padova, via Marzolo 8, 35131 Padova, Italy

${ }^{21}$ Czech Technical University, Prague, Bøehová 7, 11519 Prague 1, Czech Republic

${ }^{22}$ University of Bologna, Viale Berti Pichat 6/2, 40126 Bologna, Italy

${ }^{23}$ Department of Physics, University of Pavia, via Bassi 6, 27100 Pavia, Italy

Corresponding author: antoine.camper@cern.ch 
${ }^{24}$ The Research Council of Norway, P.O. Box 564, NO-1327 Lysaker, Norway

${ }^{25}$ Department of Physics, Heidelberg University, Im Neuenheimer Feld 226, 69120 Heidelberg, Germany

${ }^{26}$ Department of Civil Engineering, University of Brescia, via Branze 43, 25123 Brescia, Italy

\begin{abstract}
We report on recent developments in positronium work in the frame of antihydrogen production through charge exchange in the AEgIS collaboration [1]. In particular, we present a new technique based on spatially imaging a cloud of positronium by collecting the positrons emitted by photoionization. This background free diagnostic proves to be highly efficient and opens up new opportunities for spectroscopy on antimatter, control and laser manipulation of positronium clouds as well as Doppler velocimetry.
\end{abstract}

\title{
1 The baryonic asymmetry
}

The existence of antimatter has first been predicted theoretically as a solution of Paul Dirac's equation uniting quantum mechanics and relativity in 1928 [2]. Positron was the first particle of antimatter to be observed experimentally in 1932 by Anderson [3]. Since then, each fermion of the Standard model has been associated with an antimatter counterpart, which has the exact same physical properties as the matter particle but for its electrical charge being of opposite sign. When they interact, associated fundamental particles of matter and antimatter can annihilate, that is in the case of leptons, all the mass energy can be turned into gamma rays. On the other hand, each time a particle of antimatter is produced (for example by collision of particles in a collider) it is produced together with its matter counterpart. The Standard model actually predicts that as the Universe was developing, an equal amount of matter and antimatter particle should have been produced. However, the amount of antimatter observed nowadays in the Universe (in cosmic rays for example) is not anything close to matching parity with matter. This lack of antimatter is usually referred to as the baryonic asymmetry. This suggests that there might be a physical quantity accounting for this asymmetry that has not been understood yet. Solving the baryonic asymmetry requires to compare the properties of matter and antimatter with very high precision. Good candidates for precise measurements are neutral atoms less sensitive to stray magnetic and electric fields than charged particle.

At CERN, the antiproton decelerator (AD) has been producing cold (5.3 MeV) antiprotons since the year 2000 following reaction (1) happening when high energy protons are focused in an Iridium target.

$$
p+p \rightarrow p+p+p+\bar{p}
$$

In equation (1), $p$ stands for a proton and $\bar{p}$ for an antiproton. The first cold atoms of antihydrogen (the bound state of a positron and an antiproton) have been formed and observed in 2004 [4]. This colossal experimental achievement has opened up the way to compare the properties of hydrogen with those of antihydrogen, including testing the Weak Equivalence Principle (WEP). This consists in comparing the free fall of hydrogen and antihydrogen in the local gravitational field of Earth to compare the inertial and gravitational mass of the two atoms.

The AEgIS collaboration (Antihydrogen Experiment: gravity, Interferometry, Spectroscopy) is aiming at producing the first beam of cold pulsed antihydrogen in views of testing WEP on antihydrogen. AEgIS is developing an original scheme to produce 
antihydrogen which is called the charge exchange reaction [5]. This consists in transferring a positron from a positronium atom (referred to as Ps hereafter; it is the bound state of an electron and its antiparticle, the positron) towards an antiproton.

$$
P s^{*}+\bar{p} \rightarrow \bar{H}^{*}+e^{-}
$$

In equation (2), Ps* refers to a Ps atom excited in a Rydberg state, $\bar{p}$ for an antiproton, $\bar{H}^{*}$ for an atom of antihydrogen excited in a Rydberg state and $e^{-}$for an electron. The crosssection of the charge exchange reaction is highly sensitive to the temperature of both the antiproton plasma and the velocity of the Ps atoms. It is therefore important to be able to measure the temperature of the Ps cloud produced in the 1T magnetic field environment of the Penning-Malmberg trap where the antiprotons are trapped. Several difficulties have to be overcome including the limited amount of space between the Ps production target and the antiprotons trap, the low repetition rate of the pulsed Ps production and the dimensions of the magnet surrounding the antiproton trap, which makes it difficult to efficiently collect the gamma rays produced by annihilation of Ps.

We start with a presentation of the AEgIS experiment and then focus on recent advances in positronium work related to measuring the temperature of the Ps cloud used in AEgIS. In particular, we report on a new diagnostic based on a spatial imaging of the Ps cloud. In this setup, positrons photoionized from electronically excited positronium atoms are trapped along the lines of magnetic field of the trap and accelerated towards a stack of Micro Channel Plate (MCP) coupled to a phosphor screen imaged with a Charge Coupled Camera (CCD). This diagnostic is background free, single shot and can be used for spectroscopy as well as for diagnostic on the dimension of the Ps cloud and its manipulation with lasers.

\section{The AEgIS experiment}

The workhorse of the AEgIS experiment is a Penning-Malmberg trap where plasmas of antiprotons can be trapped, stored, manipulated and cooled down. A broad picture of this trap features a strong 1T magnet to trap (see Fig. 1) the antiprotons (negatively charged) on their cyclotron orbits (the magnetic field therefore prevents the antiprotons particles to escape the trap in the radial direction) and a set of electrodes shaping a potential well made of repulsive walls on both sides of the trap (the electric field therefore prevents the antiprotons to escape the trap in the longitudinal direction). The antiproton plasma trapped in this way can be cooled down by mixing with cold electrons.

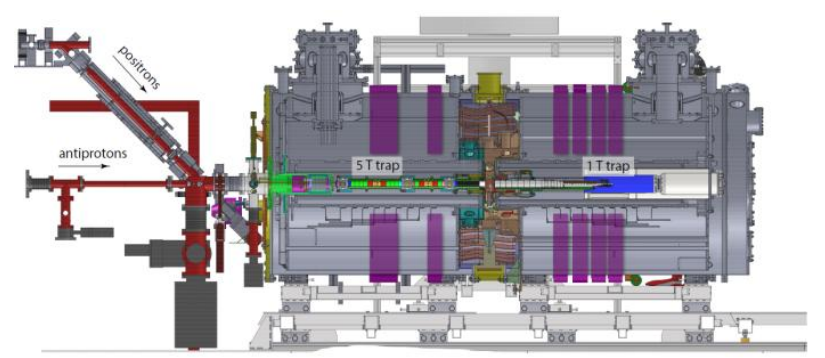

Fig. 1. Schematic of the AEgIS apparatus. Bunches of cold antiprotons are provided by the AD. The positron bunches are produced by beta decay of radioactive sodium moderated with a thin layer of solid neon and accumulated in a Surko trap. The 5T trap is used to catch the antiprotons delivered by the $\mathrm{AD}$. The $1 \mathrm{~T}$ trap is used for antihydrogen production.

The design of the electrodes used in the antihydrogen production trap further includes a $2 \mathrm{~mm}$ wide slit allowing for Ps atoms to enter the trap and interact with the antiprotons (see Fig. 2). A few millimetres above that slit, isotropic pulsed Ps clouds are produced in a nanochannel plate converter [6-9] by implanting nanosecond bunches of positrons. The 
positrons implanted on the nanochannel plate mainly fly through the silicon bulk until they reach the surface of one of the nanochannels etched in the plate. When a positron is released in the vacuum, it can bind up with an electron to form positronium. This Ps atom is formed inside a nanochannel and has to travel all the way out to the surface of the plate. In this process, Ps atoms collide many times on the walls of the nanochannel in which they were born which results into a drop in velocity. These collisions provide cooling for the Ps cloud.

As the bound state of an electron and a positron, positronium has a rather short lifetime. In its ground state with ortho spin state, this lifetime is $142 \mathrm{~ns}$. The lifetime of Ps can be extended by electronic excitation in high principal quantum number states, like Rydberg states. This also has the huge benefit of increasing the cross section of the charge exchange reaction following a $\mathrm{n}^{4}$ scaling law. In AEgIS, a two photon laser scheme [10] has been developed to produce Rydberg positronium atoms. An ultraviolet (UV, $205 \mathrm{~nm}$ ) pulse is used to excite Ps in $\mathrm{n}=3$ and a mid infrared (MIR, between $1680 \mathrm{~nm}$ and $1720 \mathrm{~nm}$ ) pulse drives the transition from $n=3$ to a Rydberg state that can be adjusted between $n=15$ and $n$ $=20$. The choice of this relatively low principal quantum number for Rydberg state is driven by the motional Stark effect (the shift of the ionization threshold due to the effective electric field experienced by an atom of Ps with a nonzero velocity component in the plane orthogonal to the magnetic field) which induces self-ionization of Ps Rydberg states with either too high velocity or too high principal quantum number state [11].

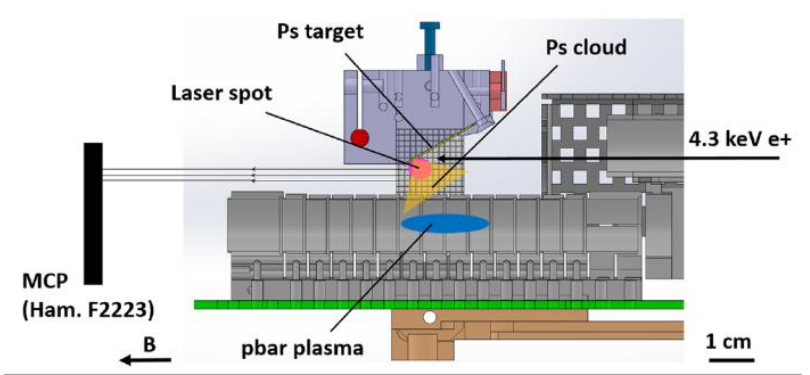

Fig. 2. Details of the antihydrogen production area. The nanochannel plate, also referred to as the Ps target, is located $5 \mathrm{~mm}$ above the trap's electrodes and can reach the antiproton plasma by diffusion through a $2 \mathrm{~mm}$ slit. Positronium is produced in reflection and the laser pulses are shining at grazing angle on the Ps target. The positrons emitted by photoionization of Ps are trapped in the lines of magnetic field and accelerated towards the MCP. A phosphor screen and CCD camera are used in association with the MCP to image the photopositrons.

The source of Ps atoms described here above is close to be isotropic and therefore most of the Ps atoms are not entering the trap because they are aiming outside the slit. In the same way, it is important to tune the laser wavelength to selectively excite the Ps atoms with velocity components aiming at the antiproton plasma. As a result, a spatially resolved tool is required to diagnose, manipulate and control the Ps cloud.

\section{A spatially resolved diagnostic for Ps}

A classical tool to study positronium is the Single-Shot Positron Annihilation Lifetime Spectroscopy (SSPALS) $[12,13]$ which consists in collecting the gamma rays emitted by annihilation of positrons with nanosecond time resolution. As this diagnostic is very useful to prove the formation of positronium and gives access to quantitative evaluation of the number of positronium atoms produced by conversion of positrons, it requires to efficiently collect the gamma rays emitted by annihilation of positrons. This includes to place detection crystals or photomultiplier close to the production area or to use very big ones. 
Also not all detectors work at cryogenic temperatures and in a strong magnetic field. The geometry of the AEgIS experiment does not allow for a highly efficient collection of the gamma rays produced by positron annihilation. In particular, the absence of free space below the target prevents long lifetime states to be revealed in the SSPALS spectrum since they annihilate in the bulk of the electrodes at about the same time as shorter lifetime states.

In order to circumvent these difficulties, we developed a new technique based on the MCP located at the very end of the apparatus (see Fig. 2) and usually used for antiproton plasma diagnostic. Just as the antiprotons are trapped on their cyclotron orbit in the Penning-Malmberg trap, electrons and positrons emitted by photoionisation in the region of the production trap will be trapped on orbits of very small diameter (less than a micron) due to the 1T magnetic field. Small orbits is the key point to achieve spatial resolution with this technique, since it forces the charged particles to keep their position with micrometric precision in the plane transverse to the magnetic field. By polarizing the front face of the MCP and applying a ramp of potential on the trap's electrodes with a slope chosen accordingly to the sign of the charged particle one wishes to image, it is possible to image the electrons emitted by photoeffect at the surface of the different elements composing the production area when they get hit by the UV laser. With settings of opposite sign, it is possible to collect the positrons emitted by photoionization of Ps. This signal is background free since there is no other source of positively charged particle in the experiment. In Fig.3, we present an image of the MCP acquired with this new diagnostic and composed as follows. In red, is an image of the trap area made visible by electrons emitted by photoeffect at the surface of the trap's electrodes (the ring in the center), the back of the Ps target (up) and other elements. This picture is superimposed with a green component which has been acquired with settings of opposite sign to collect positrons. The positrons have been emitted by photoionization of Ps atoms excited to $n=3$ with the UV pulse and further photoionised with a $1064 \mathrm{~nm}$ pulse. More precisely, what is displayed is the average over a scan of the UV wavelength throughout the Doppler profile of the Ps cloud therefore revealing the Ps cloud in its whole.

This new diagnostic is very efficient. With a single shot, it is possible to know whether the laser pulse is synchronized with the positron bunch and if the central wavelength of the UV pulse is tuned to the velocity component aiming at the antiproton plasma. When scanning the time delay between the positron cloud and the laser, one easily gets an estimate of the Ps velocity in the direction defined by the normal to the Ps target. By scanning the UV wavelength, the velocity component in the plane orthogonal to this direction can also be accessed by Doppler velocimetry. This information is required to understand the charge exchange reaction through which antihydrogen is produced and experimentally measure its cross section. The MCP diagnostic also allows the imaging of self-ionized positrons from positronium in Rydberg state submitted to the motional Stark shift. This is again very valuable in order to be able to compromise between the losses due to self-ionization and the gain stemming from the increase of cross section related to the use of higher principal quantum number state Ps atoms. 


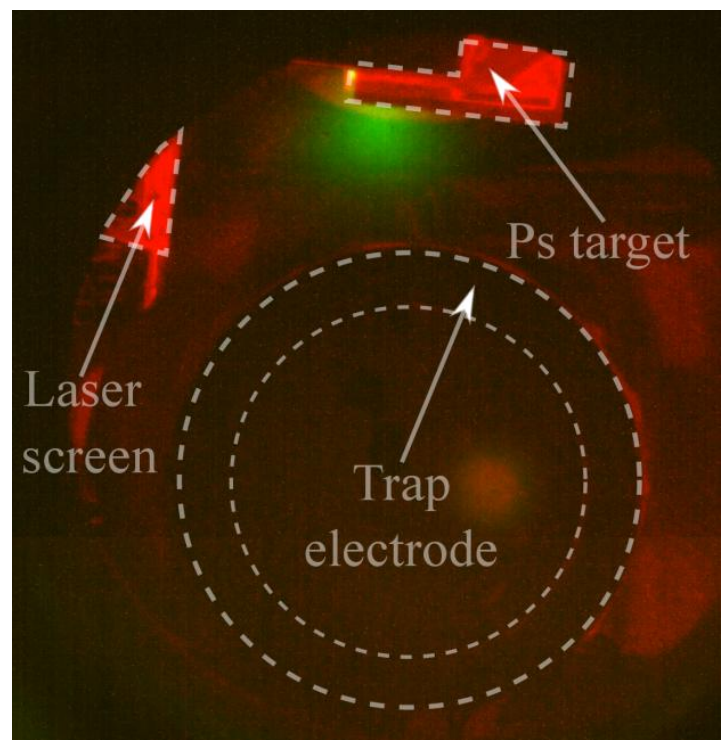

Fig. 3. Picture resulting from the superposition of two grey level images taken by the CCD camera looking at the phosphor screen of the imaging MCP. The red color channel of this picture is used to display the image that has been acquired with electrons and features the geometry of the production trap area. Semi-transparent dashed lines are used to guide the eye of the reader and help identify the elements of the production trap region. The central rings show the inner and outer edges of the circular electrodes used to trap antiprotons. The bright shape in the upper part of the picture is the bottom of the Ps target. The bright shape on the left of the picture is a screen used for laser diagnostics. The green color channel is used to display the image that has been acquired with positrons. It is actually the result of an average over a scan of the UV wavelength throughout the Doppler profile of the Ps cloud, therefore revealing the whole Ps cloud.

Doppler scans have been acquired in those conditions and show results compatible with previously reported work on Ps produced in similar conditions [14]. They are still under analysis and are therefore not presented here.

\section{Conclusion and perspectives}

We have presented the principle of the original charge exchange reaction for antihydrogen production developed in the frame of the AEgIS collaboration. We reported on a new technique allowing for efficient diagnostic and manipulation of the Ps cloud used for antihydrogen production. This scheme based on spatial imaging of charge particle opens up new opportunities for spectroscopy of antimatter including investigation of Ps Rydberg atoms self-ionisation due to motional Stark-shift in a strong magnetic field and Doppler velocimetry of a Ps cloud.

In the near future, the AD will be upgraded with a new apparatus dubbed ELENA for Extremely Low ENergy Antiproton ring which is a smaller decelerator that will provide even colder antiprotons (300 keV) starting in 2021. This will allow the different experiments taking beam from ELENA to capture more cold antiprotons and refine their work on antihydrogen.

\section{References}

1. A. Kellerbauer, et al. (AEgIS collaboration), NIM B 266, 351-356 (2008). 
2. P. Dirac, Proc. Camb. Phil. Soc. 26, 361 (1930).

3. C. Anderson, Phys. Rev. 43, 491 (1933).

4. M. Amoretti et al. (ATHENA collaboration), Nature 419, 456-459 (2002).

5. C. H. Storry et al. (ATRAP collaboration) Phys. Rev. Lett. 93, 263401 (2004).

6. S. Mariazzi, P. Bettotti, and R. S. Brusa, Phys. Rev. Lett. 104, 243401 (2010).

7. S. Mariazzi, P. Bettoni, S. Larcheri, L. Toniutti, and R. S. Brusa, Phys. Rev. B, 81, 235418 (2010).

8. S. Mariazzi, A. Salemi, and R. S. Brusa, Phys. Rev. B, 78 (2008).

9. S. Aghion et al. (AEgIS collaboration), NIM B 362, 86-92 (2015).

10. S. Cialdi, I. Boscolo, F. Castelli, F. Villa, G. Ferrari, M. Giammarchi, NIM B 269, 1527-1533 (20).

11. D. B. Cassidy, Eur. Phys. J. D 72, 53 (2018).

12. S. Aghion et al. (AEgIS collaboration), Phys. Rev. A 94, 012507 (2016).

13. S. Aghion et al. (AEgIS collaboration), Phys. Rev. A 94, 013402 (2018).

14. R. S. Vallery, P. W. Zitzewitz, and D. W. Gidley, Phys. Rev. Lett. 90, 203402 (2003). 